\title{
Rehashing Trial Results Won't Help with Puzzling Aneurysms- Patients Need Best Care within a Contemporary Trial
}

W e would like to air our concerns regarding 2 recent publications in the American Journal of Neuroradiology: a metaanalysis of coil embolization versus clipping for ruptured aneurysms ${ }^{1}$ and the accompanying editorial entitled "Best Evidence: Comments on Meta-Analysis of Coiling versus Clipping," by Sellar and White. ${ }^{2}$

Let us begin with the original research paper. The authors admit conflating the results of 2 randomized trials (the Kuopio study $^{3}$ and the International Subarachnoid Aneurysm Trial $[\mathrm{ISAT}]^{4}$ ) with a study that used a different design (the Barrow Ruptured Aneurysm Trial [BRAT] ${ }^{5}$ ). Whether it is valid to "average " the results of randomized trials with those of a prerandomized trial such as BRAT (with a high rate of cross-overs from coiling to clipping) in a meta-analysis remains unclear. Because they chose not to follow the Preferred Reporting Items for Systematic Reviews and Meta-Analyses (PRISMA) guidelines for reporting meta-analyses, ${ }^{6}$ the scientific appraisal of their report is problematic.

For this to be followed by an editorial entitled "Best Evidence" seems to be overstating the case. Skepticism, the mother of science, tells us not to uncritically accept those opinions that just happen to correspond with our own. One all-too-common problem with the interpretation of scientific studies is the extrapolation of findings to patients who were not included in the original studies. The sweeping application of ISAT results to all aneurysms is a good example of this problem. There can be no support, statistical or other, for such inferences. ${ }^{7}$

The authors of the editorial failed to make important distinctions: the free statement "unequivocal evidence exists that the results of coiling are superior to those of clipping in the treatment of aneurysms" is a sweeping statement, unjustified by evidence.

First, the editorialists failed to distinguish ruptured from unruptured aneurysms, whereas there are many reasons to suspect that treatment of the 2 conditions may differ. Surgical clipping of unruptured intracranial aneurysms may very well be superior to coiling, for all we know, at the time of writing this comment. The best treatment of unruptured aneurysms

http://dx.doi.org/10.3174/ajnr.A3652 remains to be seen, and a trial addressing the question has been launched. ${ }^{8}$

Second, Lanzino et $\mathrm{al}^{1}$ mentioned, but the authors of the editorial failed to emphasize, that the Kuopio and ISAT populations were only a small portion of all patients with ruptured aneurysms. This does not mean these trials were biased, as is often claimed in the surgical literature, ${ }^{9}$ only that it affects the generalizability of the study results to future patients. In the BRAT, a study initially designed to palliate this problem and to include all aneurysms, a significant proportion of patients allocated to coiling were crossed over to clipping. If the "intent to coil " BRAT group results were in line with ISAT results, almost $40 \%$ of patients of that group were deemed to be better treated surgically, for reasons of "clinical judgment" that remain difficult to specify. It is important to emphasize that we do not know for sure that clipping was best for the patients who were crossed over. These patients were included in the analysis, but no one knows for sure what the best treatment for these cases was, or is.

One important point is that ISAT, a trial interrupted more than 10 years ago, weighs heavily in the overall results of the metaanalysis. Who were these patients in ISAT? They were good-grade patients bearing small anterior circulation ruptured aneurysms with anatomic characteristics favorable for coiling. The small amount of proof we have won in our field is supported by a single large, properly conducted trial, which showed that for patients with aneurysmal subarachnoid hemorrhage for whom coiling and clipping were considered good options 10-18 years ago, random allocation to coiling led to better outcomes compared with surgery.

Many patients are still offered clipping despite ISAT results, but there are many more patients who today are only offered coiling, though they would not have been included in the original ISAT study. Admittedly, and, as mentioned by Sellar and White, ${ }^{2}$ technical developments now permit endovascular treatment for aneurysms that decades ago would not have been considered for coiling. Again, no one knows for sure if these patients would not be better served with a surgical approach. However, rather than threatening clinicians who consider clipping for some cases to be at risk of litigation or suggesting that the expert opinion of a coiler could protect them from such litigation, we propose that we have yet to complete the task of gathering the evidence for all patients 
not included in the original ISAT study. For patients with ruptured aneurysms for whom we still do not know what is best between clipping and coiling, optimal treatment is to offer a trial comparing the 2 treatment options. For those who properly remain uncertain, ISAT-II is underway. ${ }^{10}$

\section{REFERENCES}

1. Lanzino G, Murad MH, d'Urso PI, et al. Coil embolization versus clipping for ruptured intracranial aneurysms: a meta-analysis of prospective controlled published studies. AJNR Am J Neuroradiol 2013 Apr. 11. [Epub ahead of print]

2. Sellar RJ, White P. Best evidence: comments on meta-analysis of coiling versus clipping. AJNR Am J Neuroradiol 2013 Apr 4. [Epub ahead of print]

3. Koivisto T, Vanninen R, Hurskainen H, et al. Outcomes of early endovascular versus surgical treatment of ruptured cerebral aneurysms: a prospective randomized study. Stroke 2000;31:2369-77

4. Molyneux A, Kerr R, Stratton I, et al. International Subarachnoid Aneurysm Trial (ISAT) of neurosurgical clipping versus endovascular coiling in 2143 patients with ruptured intracranial aneurysms: a randomised trial. Lancet 2002;360:1267-74

5. McDougall CG, Spetzler RF, Zabramski JM, et al. The Barrow Ruptured Aneurysm Trial. J Neurosurg 2012;116:135-44
6. Moher D, Liberati A, Tetzlaff J, et al, the PRISMA Group (2009). Preferred Reporting Items for Systematic Reviews and MetaAnalyses: the PRISMA Statement. BMJ 2009;339:b2535

7. Ludbrook J. Analysis of $2 \times 2$ tables of frequencies: matching test to experimental design. Int J Epidemiol 2008;37:1430-35

8. Darsaut TE, Findlay JM, Raymond J, CURES Collaborative Group. The design of the Canadian UnRuptured Endovascular versus Surgery (CURES) trial. Can J Neurol Sci 2011;38:236-41

9. Raymond J, Kotowski M, Darsaut TE, et al. Ruptured aneurysms and the International Subarachnoid Aneurysm Trial (ISAT): what is known and what remains to be questioned. Neurochirurgie 2012;58:103-08

10. Darsaut TE, Jack A, Kerr RS, et al, for the ISAT-II Collaborative Group. International subarachnoid aneurysm trial - ISAT Part II: study protocol for a randomized controlled trial. Trials 2013;14:156

T.E. Darsaut

University of Alberta Hospital Mackenzie Health Sciences Centre Division of Neurosurgery, Department of Surgery Edmonton, Alberta, Canada J. Raymond Centre Hospitalier de l'Université de Montréal Notre-Dame Hospital Department of Radiology Montreal, Quebec, Canada 